\title{
Dane Particles in the Sera of Patients with Chronic Aggressive Hepatitis
}

\author{
Gotaro Yamada, Toshinari Kobayashi, Takao Tsuji, \\ Yasuyuki Ohta, Kiyowo Kosaka \\ The First Department of Internal Medicine, Okayama University \\ Medical School, Okayama
}

Yamada, G., Kobayash, T., Tsuji, T., Ohta, Y. and Kosaka, K. Dane Particles in the Sera of Patients with Chronic Aggressive Hepatitis. Tohoku J. exp. Med., 1973, 111 (1), 93-95 — Dane particles were observed electron microscopically in sera of all 10 patients with Australia ( $\mathrm{Au}$ ) antigen-positive chronic aggressive hepatitis. Massive Dane particles were demonstrated in sera of a patient at the early phase of the elevation of serum glutamic pyruvic transaminase. The inner body of a Dane particle was shown as a typical icosahedral symmetry by rotation technique. - Au antigen; HB antigen; Dane particle; chronic aggressive hepatitis

Dane particles (Dane et al. 1970) (42 nm particles) have been observed together with $20 \mathrm{~nm}$ spherical particles and tubular forms in Australia (Au) antigen-antibody complexes in sera of patients with $\mathrm{Au}$ antigen-positive hepatitis (Almeida and Waterson 1969; Field and Cossart 1971), but little is known about the number of Dane particles in the complexes before and after liver cell damage as indicated by a raised serum glutamic pyruvic transaminase (SGPT).

We have studied sera of 10 patients with $\mathrm{Au}$ antigen-positive chronic hepatitis once a week or a month for up to 6 months. Histologically all cases were proved to be chronic aggressive hepatitis of the severe form. A modified method of Almeida and Waterson

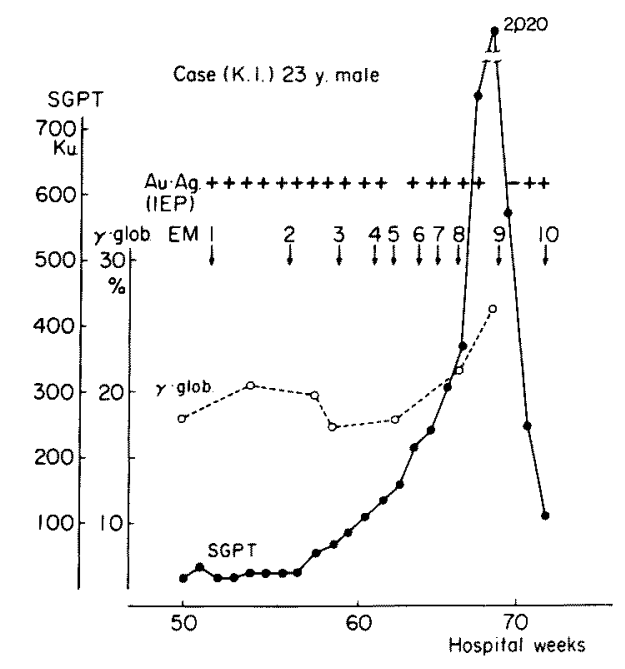

Fig. 1. A 23-year-old male with chronic aggressive hepatitis. Massive appearance of Dane particles in EM 6-8. Large complexes composed mainly of $20 \mathrm{~nm}$ particles are observed in EM 9. ("EM 1" denotes first observation by electron microscopy).

Received for publication, February 1, 1973. 
(1969) was used for electron microscopy; one $\mathrm{ml}$ of whole serum was spun at $23,000 \times \mathrm{g}$ for 1 hour, and pellet was washed twice in phosphate-buffered saline ( $\mathrm{pH} \mathrm{7.2)}$ at a similar speed. Specimens were negatively stained with $2 \%$ phosphotungstic acid at $\mathrm{pH} 6.5$ and examined under an HU-12 electron microscope.

In all cases, the groups composed of large Au antigen-antibody complexes were observed transiently or persistently during electron microscopic observations. Dane particles were found in tho complexes of all cases, but in 7 of 10 cases they were only under a few per cents among all $\mathrm{Au}$ particles. In 2 of the remaining 3 cases large particles were constantly observed at the rate of 10-20 per cents independently of a elevation of SGPT. We have found the last case whose sera contained a large number of Dane particles transiently at the exrly phase of the elevation of SGPT up to 2,020 Karmen units (Fig. 1). During the period of EM 1-5 in Fig. 1, a few Dane particles were observed among partly aggregated and partly scattered $20 \mathrm{~nm}$ particles and tubular forms ("EM 1" denotes the first electron

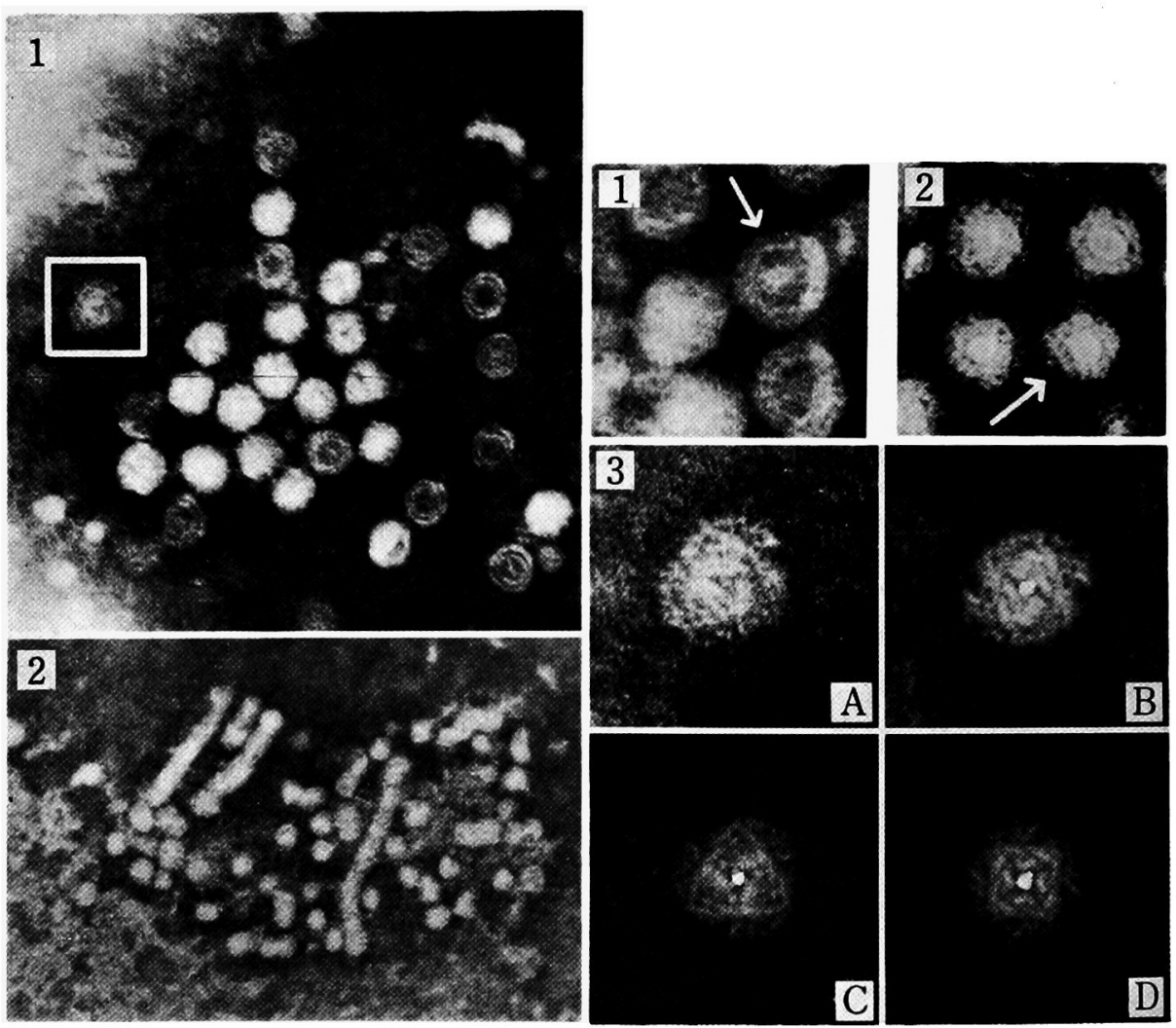

Fig. 2-1, Aggregated and free Dane particles are observed at EM 8 in Fig. $1(\times 120,000)$.

2-2 Small aggregates of $20 \mathrm{~nm}$ particles and tubular forms are observed at EM 10 in Fig. $1(\times 120,000)$.

Fig. 3-1. An arrow shows a particle with a suggestive hexagonal inner body $(\times 270,000)$.

3-2 An arrow shows a particle with a suggestive pentagonal inner body $(\times 270.000)$.

3-3 A Dane particle obtained by the rotation method showing the structure of an icosahedral symmetry $(\times 270,000)$.

A. The original electron micrograph indicated by the square in Fig. 2-1.

B. Rotated $\mathrm{n}=\mathbf{2}$.

C. Rotated $n=3$, showing a typical icosahedral symmetry.

D. Rotated $n=4$. 
microscopic observation). On the other hand, in the period of EM 6-8, a large number of aggregated and free Dane particles were observed. At the time of a maximum elevation of SGPT (EM 9), Dane particles were hardly found, whereas large complexes composed mainly of small spherical particles were observed. One month later (EM 10), the large complexes as mentioned above were no more found and the patient serum contained small aggregates of $20 \mathrm{~nm}$ particles and tubular forms (Fig. 2-2).

Fig. 2-1 shows aggregated and free Dane particles at the time of EM 8. One can see an internal structure in about half of the particles; "the inner bodies" (Dane et al. 1970) are observed in these particles. Some inner bodies appear to have 5 or 6 facets in symmetry (Fig. 3-1, 2), as Dane et al. (1970) suggested. Using the rotation method (Markham et al. 1963), the inner body of a particle with a suggestive icosahedral symmetry proved to have a typical symmetry (Fig. 3-3).

It is conceivable that the transiently appeared massive Dane particles may be related with liver cell damage as indicated by a raised SGPT in this case.

\section{Acknowledgment}

We wish to express our thanks to Prof. N. Ishida of the Tohoku University School of Medicine for his interest and guidance in this investigation.

\section{References}

1) Almeida, J.D. \& Waterson, A.P. (1969) Immune complexes in hepatitis. Lancet, 2, 983-986.

2) Dane, D.S., Cameron, C.H. \& Briggs, M. (1970) Virus-like particles in serum of patients with Australia-antigen-associated hepatitis. Lancet, 1, 695-698.

3) Field, A.M. \& Cossart, Y.E. (1971) Specificity of Au antibodies. Lancet, 2, 91.

4) Markham, R., Frey, S. \& Hills, G.J. (1963) Methods for the enhancement of image detail and accentuation of structure in electron microscopy. Virology, 20, 88-102. 